\title{
Super-selective ASL and 4D ASL-based MR Angiography in a Patient with Moyamoya Disease
}

\section{Case Report}

\author{
Nico Sollmann ${ }^{1,2}$ (D) Hans Liebl ${ }^{1} \cdot$ Christine Preibisch ${ }^{1,2} \cdot$ Claus Zimmer $^{1,2} \cdot$ Michael Helle $^{3} \cdot$ Makoto Obara4 $^{4}$ \\ Jan S. Kirschke ${ }^{1,2} \cdot$ Stephan Kaczmarz ${ }^{1,2}$
}

Received: 31 July 2020 / Accepted: 27 August 2020 / Published online: 25 September 2020

(c) The Author(s) 2020

$\begin{array}{ll}\text { Abbreviations } \\ \text { ASL } & \begin{array}{l}\text { Arterial spin labeling } \\ \text { CENTRA }\end{array} \\ \begin{array}{l}\text { Contrast-enhanced timing-robust } \\ \text { phy }\end{array} \\ \text { CT } & \text { Compiogra- } \\ \text { DSA } & \text { Digital subtraction angiography } \\ \text { DSC } & \text { Dynamic susceptibility contrast } \\ \text { EPI } & \text { Echo planar imaging } \\ \text { FLAIR } & \text { Fluid-attenuated inversion recovery } \\ \text { GRE } & \text { Gradient recalled echo } \\ \text { ICA } & \text { Internal carotid artery } \\ \text { MRA } & \text { Magnetic resonance angiography } \\ \text { MRI } & \text { Magnetic resonance imaging } \\ \text { TOF } & \text { Time-of-flight }\end{array}$

The authors Nico Sollmann and Hans Liebl contributed equally to the manuscript.

\author{
Nico Sollmann \\ Nico.Sollmann@tum.de \\ Hans Liebl \\ Hans.Liebl@tum.de \\ Christine Preibisch \\ Preibisch@tum.de \\ Claus Zimmer \\ Claus.Zimmer@tum.de \\ Michael Helle \\ mhelle@web.de \\ Makoto Obara \\ Makoto.Obara@philips.com
}

\section{Introduction}

Moyamoya disease is a cerebrovascular disorder with progressive steno-occlusive affection of mainly the bilateral intracranial internal carotid artery (ICA) and its proximal branches [1,2]. Moyamoya disease is predominantly observed in Japan, whereas the incidence in Europe is low and appears to amount to only one tenth of that observed in Japan [1,3]. When moyamoya disease becomes clinically manifest, it entails symptoms due to brain ischemia (e.g., stroke) and/or due to compensatory mechanisms in response to ischemia (e.g., hemorrhage from fragile collateral vessels) [1].

Digital subtraction angiography (DSA) is the standard of reference for the diagnosis of moyamoya disease [1, 4]; however, computed tomography (CT) or, when rapidly available, magnetic resonance imaging (MRI) are the first-

Jan S. Kirschke

Jan.Kirschke@tum.de

Stephan Kaczmarz

Stephan.Kaczmarz@tum.de

1 Department of Diagnostic and Interventional Neuroradiology, Klinikum rechts der Isar, Technische Universität München, Ismaninger Str. 22, 81675 Munich, Germany

2 TUM-Neuroimaging Center, Klinikum rechts der Isar, Technische Universität München, Munich, Germany

3 Department of Radiology and Neuroradiology, University Hospital Schleswig-Holstein, Campus Kiel, Arnold-Heller-Str. 3, 24103 Kiel, Germany

4 Philips Electronics Japan Healthcare, 13-37, Kohnan 2-chome, Tokyo 108-8507, Japan 
Fig. 1 Fluid-attenuated inversion recovery (FLAIR; a) combined with diffusion-weighted imaging (b) and corresponding apparent diffusion coefficient map displayed in (c) revealed a subacute postischemic lesion in the left-hemispheric white matter. Time-of-flight (TOF) magnetic resonance angiography (MRA; d) showed multiple changes of arterial vascular calibers (with considerably reduced flow signals of the bilateral internal carotid artery [ICA] and its proximal branches)
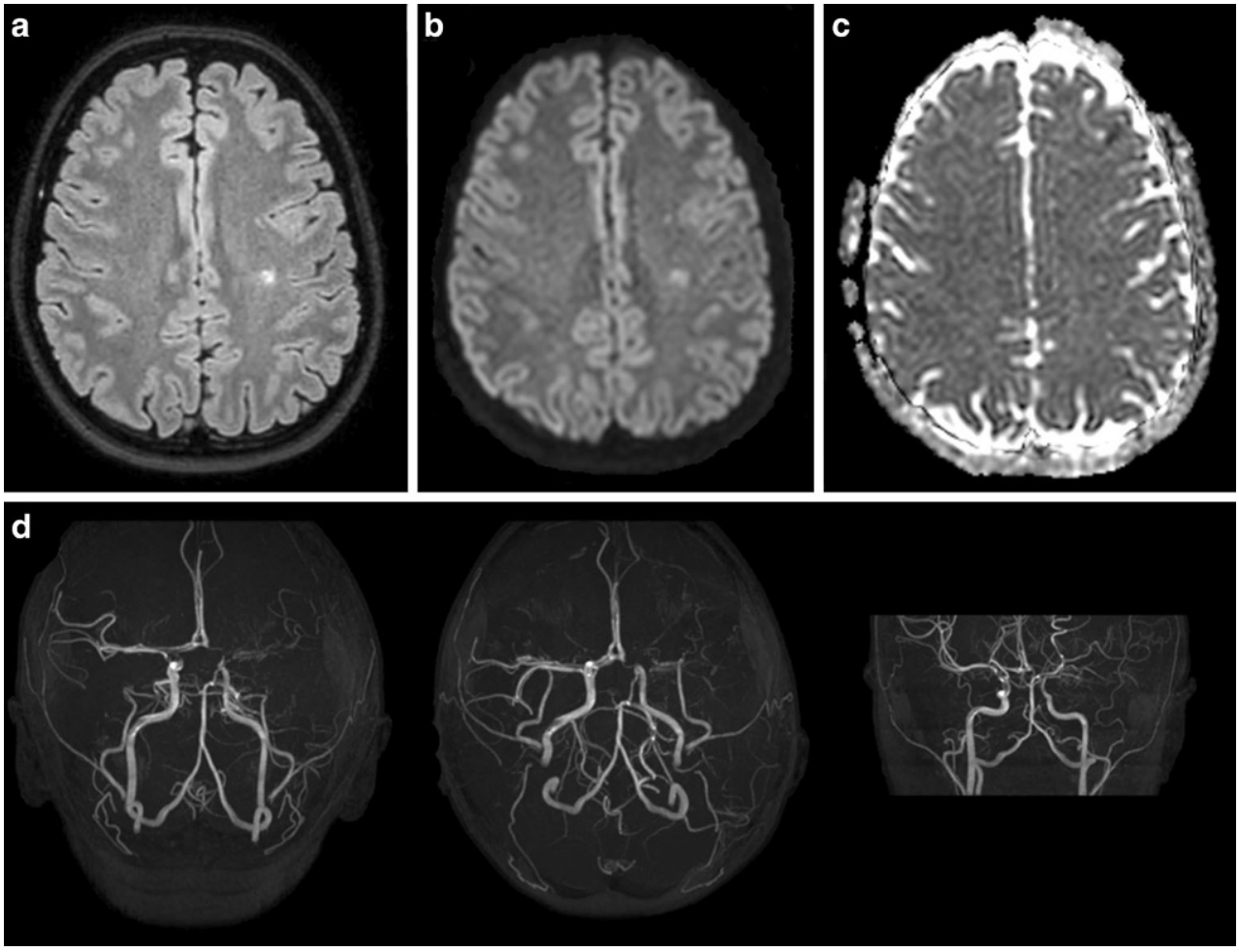

line modalities when a patient with neurological symptoms, potentially attributable to moyamoya disease, is referred for diagnosis. Time-of-flight (TOF) magnetic resonance angiography (MRA) is commonly used as the sequence of choice for evaluation of steno-occlusive vascular changes; however, it is static and cannot capture dynamic flow information, neither does it provide information about the potentially complex intracranial perfusion changes related to collateral vessels in moyamoya disease.

This article presents an imaging approach including super-selective arterial spin labeling (ASL) and super-selective 4D ASL-based MRA to non-invasively capture hemodynamic changes and time-resolved vessel architecture with a clinically applicable scan protocol in a patient with moyamoya disease.

\section{Case Presentation}

\section{Clinical Symptoms}

A 26-year-old German woman reported fluctuating intermittent hemihypesthesia of the right side of the body with acute onset. The patient did not show any focal neurological deficits during clinical examination. Her family anamnesis did not reveal any prior ischemic events, and migraine was the only known pre-existing condition.

\section{Magnetic Resonance Imaging}

The patient was referred for MRI to screen for structural cerebral pathology, with the suspected diagnosis of ischemic stroke according to the neurological report at admission. Scanning was performed on a 3-Tesla system (Achieva dStream, software release R5.6; Philips Healthcare, Best, The Netherlands) using a 32-channel head coil and a custom patch (to facilitate super-selective imaging).

The standard protocol included a 3D fluid-attenuated inversion recovery (FLAIR), TOF-MRA, diffusion-weighted imaging, and $\mathrm{T} 2 *$-weighted sequence. The protocol revealed a FLAIR hyperintense (Fig. 1a) lesion in the lefthemispheric white matter (affecting the precentral and postcentral gyrus), which showed residual restricted diffusion (Fig. 1b,c), indicating subacute ischemia. In addition, TOFMRA revealed multiple irregularities of arterial vascular calibers, most prominently captured by reduced flow signals of the bilateral ICA and its proximal branches (with the left-sided distal ICA showing discontinuous flow signal, indicative of pseudo-occlusive stenosis; Fig. 1d).

Due to these findings, non-selective pseudocontinuous ASL was applied according to the latest recommendations [5] (with manually set labeling plane based on phase contrast angiography, $1800 \mathrm{~ms}$ label duration, $2000 \mathrm{~ms}$ postlabel delay, 2D gradient recalled echo [GRE] echo planar imaging [EPI] readout, TR/TE/alpha $=4500 \mathrm{~ms} / 9.98 \mathrm{~ms} / 90^{\circ}$, $3.3 \times 3.6 \times 6 \mathrm{~mm}^{3}$ spatial resolution, $0.6 \mathrm{~mm}$ gap, 16 slices, 
Fig. 2 Pseudocontinuous arterial spin labeling (ASL) showed mostly normal cerebral blood flow (a; in $\mathrm{ml} / 100 \mathrm{~g} / \mathrm{min})$, super-selective ASL showed reduced but detectable supply of the left middle cerebral artery territory by the left-sided internal carotid artery (ICA; b) and complete supply of the left anterior cerebral artery territory by the contralateral ICA (two representative axial slices; $\mathbf{c})$
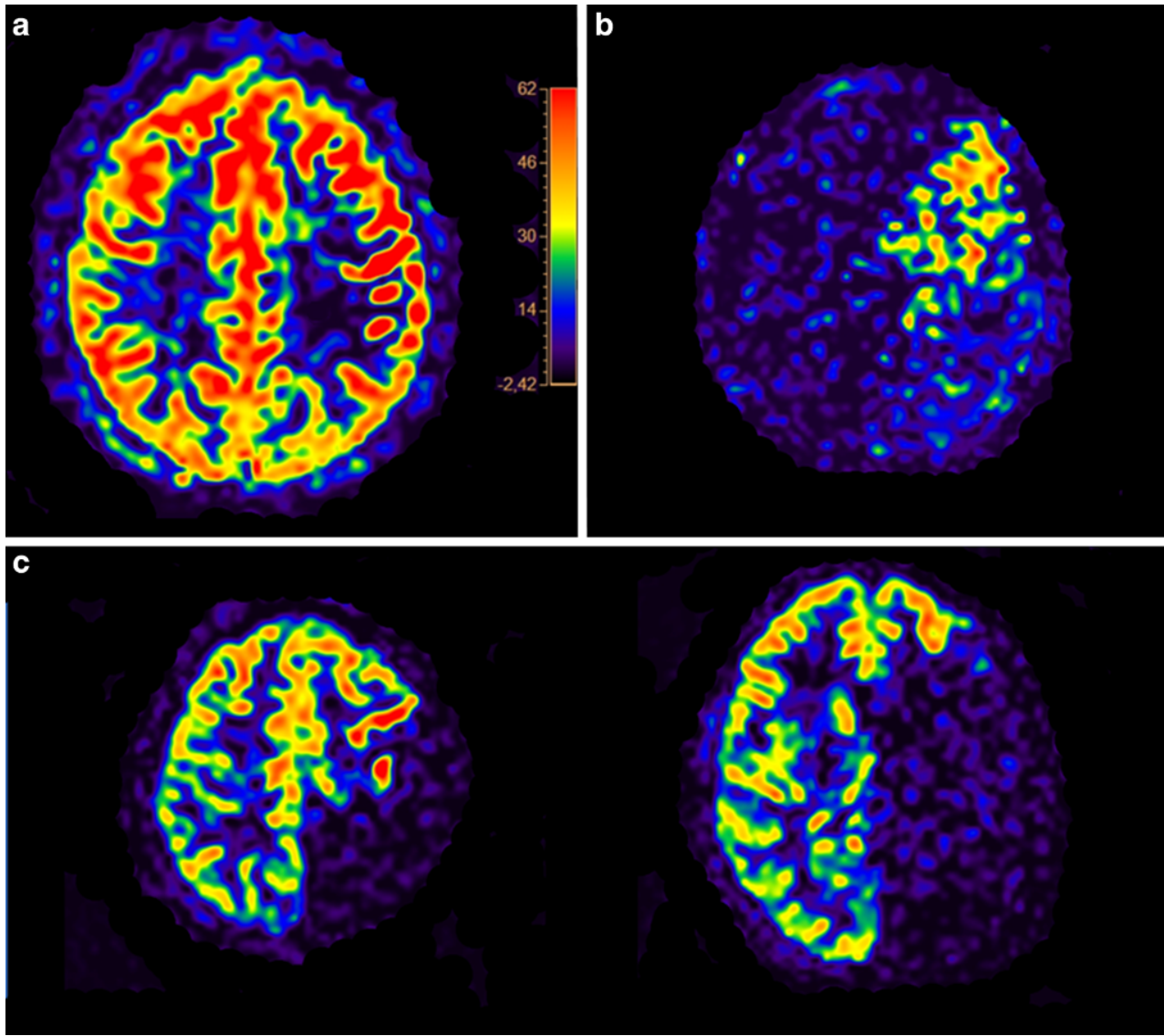

background suppression, EPI factor 29, scan time: $4 \mathrm{~min}$ $48 \mathrm{~s}$ ). Furthermore, super-selective ASL (according to Helle et al. [6]; similar parameters as for pseudocontinuous ASL with $1800 \mathrm{~ms}$ post-label delay, scan time: $4 \mathrm{~min} 3 \mathrm{~s}$ ) and 4D MRA based on super-selective ASL with contrastenhanced timing-robust angiography (CENTRA) keyhole and view-sharing technique (4D-PACK according to Togao et al. [7]; 3D GRE readout, 7 time points at 100, 200, 500, $800,1200,1600$ and $2000 \mathrm{~ms}$, turbo factor 60 , TR/TE/ alpha $=4982 \mathrm{~ms} / 1.7 \mathrm{~ms} / 11^{\circ}, 1 \times 1.3 \times 1.6 \mathrm{~mm}^{3}$ spatial resolution, scan time: $5 \mathrm{~min} 10 \mathrm{~s}$ ) were separately applied for the left and right ICA. Labeling positions of super-selective acquisitions were successfully set fully automated based on TOF-MRA of the neck arteries $\left(1.5 \times 1.5 \times 2 \mathrm{~mm}^{3}\right.$ spatial resolution, acquired within $45 \mathrm{~s}$ with Compressed SENSE) by an additional software tool integrated in the scanner software.

Pseudocontinuous ASL revealed mostly normal cerebral blood flow globally (Fig. 2a), super-selective ASL was indicative of reduced but detectable supply of the left middle cerebral artery territory by the left-sided ICA and complete supply of the left anterior cerebral artery territory by the contralateral ICA (Fig. 2b,c). The super-selective 4D ASL-based MRA was able to capture the aberrant interhemispheric supply patterns in a time-resolved manner (takeover of the left anterior cerebral artery territory by branches of the contralateral hemisphere and prominent left-sided communicating posterior artery with retrograde filling of branches of the middle and anterior cerebral arteries; Fig. 3a, b). Furthermore, collaterals indicative of moyamoya disease were observed, most prominently originating around the distal left-sided ICA and its proximal branches (Fig. 3b). The super-selective 4D ASL-based MRA showed high signal intensity and enabled depiction of distal arterial branches (Fig. 3a, b).

To rule out vasculitis, additional non-contrast-enhanced and contrast-enhanced T1-weighted black blood sequences were acquired, which did not show any vessel wall contrast enhancement. Furthermore, dynamic susceptibility contrast (DSC) perfusion (dynamic acquisition of 80 gradient-echo EPI volumes, TR/TE/alpha $=1483 \mathrm{~ms} / 30 \mathrm{~ms} / 60^{\circ}, 26$ slices, $2.0 \times 2.0 \times 3.5 \mathrm{~mm}^{3}$ spatial resolution, acquired within $2 \mathrm{~min}$ $3 \mathrm{~s}$ during injection of a weight-adjusted Gd-DOTA bolus) showed an increased time to maximum and mean transit time (Fig. 4a-d).

\section{Digital Subtraction Angiography}

Two days after MRI acquisition, DSA of the brain-feeding arteries was performed (Azurion; Philips Healthcare, Best, 
Fig. 3 Super-selective 4D arterial spin labeling (ASL)-based magnetic resonance angiography (MRA; representative images with a delay of $2000 \mathrm{~ms}$ ) and digital subtraction angiography (DSA) of the right-sided internal carotid artery (ICA; a) and left-sided ICA (b) showed aberrant interhemispheric supply patterns in time-resolved manner (takeover of the left anterior cerebral artery territory by branches of the contralateral hemisphere and prominent leftsided communicating posterior artery with retrograde filling of branches of the middle and anterior cerebral artery). Collaterals indicative of moyamoya disease were observed, primarily around the distal left-sided ICA and its proximal branches
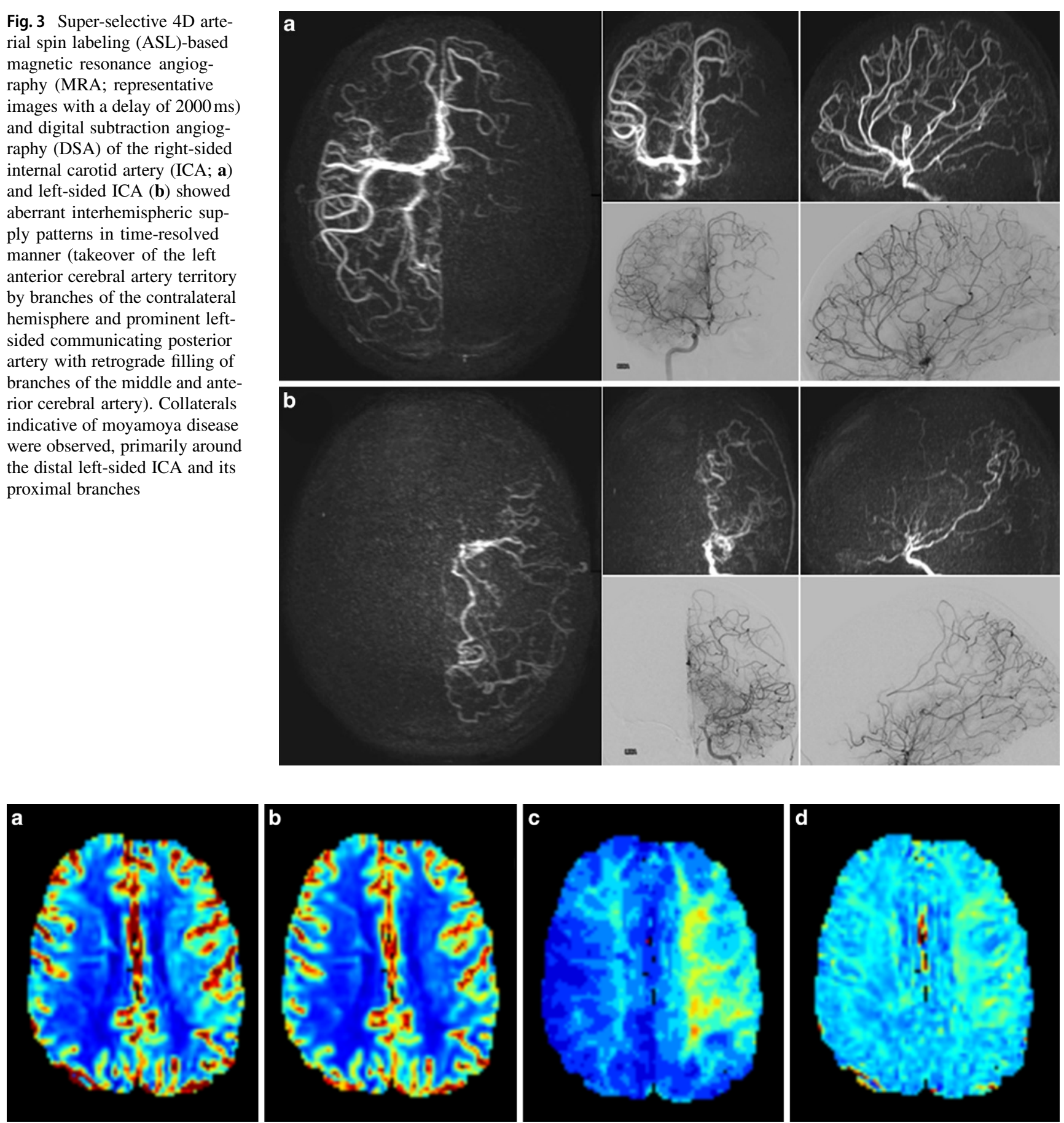

Fig. 4 Dynamic susceptibility contrast (DSC) perfusion showing mostly normal to slighlty elevated cerebral blood volume (a) and cerebral blood flow (b), combined with an increased time to maximum (c) and mean transit time (d) for the left hemisphere

The Netherlands). Coronal and lateral views were obtained after the injection of a bolus of iodinated contrast agent in the ICA and vertebral arteries of both sides. Very good visual concordance was found between images of the superselective 4D ASL-based MRA and DSA (Fig. 3a, b).

\section{Discussion}

In MRI of suspected cerebrovascular pathology, TOF-MRA is used for evaluation of steno-occlusive vascular changes during clinical routine. However, this sequence lacks reliability in visualizing slow blood flow or flow that is not directed in the feet-to-head direction, and it cannot pro- 
vide dynamic flow information since it is a static technique. Hence, in disorders with changed brain perfusion patterns and development of collateral circulations, such as moyamoya disease, it may be of limited value.

In the presented patient case, a super-selective 4D ASLbased MRA was able to reveal time-resolved flow signals of the distal ICA that could predict the territorial perfusion and collateral vessels confirmed by DSA performed during the later course. Thus, prior to DSA, complete occlusion of the left-sided ICA could be ruled out as part of the primary diagnostic MRI examination, simultaneously displaying peripheral intracranial arterial branches as well as the formed collateral vessels characteristic of moyamoya disease. Of note, using super-selective ASL investigations of hemodynamic changes as well as for the depiction of the altered vessel architecture seem feasible as part of routine clinical MRI.

To the authors' knowledge, the acquired 4D ASL-based MRA, referred to as 4D-PACK, has been used by only two preceding investigations $[7,8]$; yet, in these two publications, no super-selective acquisitions were performed. One comparative study in Japanese patients with moyamoya disease investigated vessel visualization by the technique in relation to inflow-enhanced multi-phase angiography and DSA, pointing at significantly improved visualization of cerebral arteries and leptomeningeal collaterals for non-selective 4D-PACK [7]. High arterial signal in time-resolved manner can be obtained even at late time points because the sequence is based on pseudocontinuous ASL instead of pulsed ASL, and scanning is accelerated using the keyhole and view-sharing techniques [7, 8]. Thus, obtaining data for multiple post-labeling time points with clinically acceptable acquisition times becomes possible while achieving high spatial resolution with sufficient anatomical coverage and high vessel signal. However, the previous work did not apply the sequence in the acute setting, as done in the present investigation with very unclear blood supply patterns due to largely missing flow signal as depicted by TOF-MRA of the distal ICA, nor did it achieve a combination with superselective ASL. In addition, the successfully applied novel automated labeling approach may particularly increase the feasibility in clinical routine, circumventing the need for time-consuming and sometimes error-prone manual labeling by specifically trained personal.

\section{Conclusion}

The combination of super-selective ASL and super-selective 4D ASL-based MRA with automatic labeling presents a promising approach to evaluate hemodynamic changes and vessel architecture in a time-resolved manner with high resolution. The approach seems highly feasible for clinical routine MRI scan protocols. The super-selective 4D ASLbased MRA sequence allows immediate evaluation of selected arterial branches and collateral vessels in cerebrovascular diseases before DSA is performed, which is achieved without the need to apply a contrast agent.

Acknowledgements We thank Kim van de Ven (Philips Healthcare, Best, The Netherlands) for support with ASL imaging.

Funding Open Access funding provided by Projekt DEAL.

Conflict of interest M. Obara is an employee of Philips Electronics Japan Healthcare. N. Sollmann, H. Liebl, C. Preibisch, C. Zimmer, M. Helle, J.S. Kirschke and S. Kaczmarz declare that they have no competing interests.

Open Access This article is licensed under a Creative Commons Attribution 4.0 International License, which permits use, sharing, adaptation, distribution and reproduction in any medium or format, as long as you give appropriate credit to the original author(s) and the source, provide a link to the Creative Commons licence, and indicate if changes were made. The images or other third party material in this article are included in the article's Creative Commons licence, unless indicated otherwise in a credit line to the material. If material is not included in the article's Creative Commons licence and your intended use is not permitted by statutory regulation or exceeds the permitted use, you will need to obtain permission directly from the copyright holder. To view a copy of this licence, visit http://creativecommons.org/licenses/by/4. $0 /$

\section{References}

1. Scott RM, Smith ER. Moyamoya disease and moyamoya syndrome. N Engl J Med. 2009;360:1226-37.

2. Suzuki J, Kodama N. Moyamoya disease-a review. Stroke. 1983; 14:104-9.

3. Yonekawa Y, Ogata N, Kaku Y, Taub E, Imhof HG. Moyamoya disease in Europe, past and present status. Clin Neurol Neurosurg. 1997;99 Suppl 2:S58-60.

4. Hasuo K, Tamura S, Kudo S, Uchino A, Carlos R, Matsushima T, Kurokawa T, Kitamura K, Matsuura K. Moya moya disease: use of digital subtraction angiography in its diagnosis. Radiology. 1985; 157:107-11.

5. Alsop DC, Detre JA, Golay X, Günther M, Hendrikse J, Hernandez-Garcia L, Lu H, MacIntosh BJ, Parkes LM, Smits M, van Osch MJ, Wang DJ, Wong EC, Zaharchuk G. Recommended implementation of arterial spin-labeled perfusion MRI for clinical applications: A consensus of the ISMRM perfusion study group and the European consortium for ASL in dementia. Magn Reson Med. 2015;73:102-16.

6. Helle M, Norris DG, Rüfer S, Alfke K, Jansen O, van Osch MJ. Superselective pseudocontinuous arterial spin labeling. Magn Reson Med. 2010;64:777-86.

7. Togao O, Hiwatashi A, Obara M, Yamashita K, Momosaka D, Nishimura A, Arimura K, Hata N, Yoshimoto K, Iihara K, Van Cauteren M, Honda H. 4D ASL-based MR angiography for visualization of distal arteries and leptomeningeal collateral vessels in moyamoya disease: a comparison of techniques. Eur Radiol. 2018;28:4871-81.

8. Obara M, Togao O, Beck GM, Shibukawa S, Okuaki T, Yoneyama M, Nakamura M, Honda H, Van Cauteren M. Non-contrast enhanced 4D intracranial MR angiography based on pseudo-continuous arterial spin labeling with the keyhole and view-sharing technique. Magn Reson Med. 2018;80:719-25. 